\title{
Efecto de una Intervención Educativa vía WhatsApp en la Higiene Oral de Pacientes con Ortodoncia
}

\author{
Effect of an Educational Intervention via WhatsApp \\ on the Oral Hygiene of Orthodontic Patients
}

Creysi Puyén Goicochea'; Johan Armas Pérez' \& Mariano Ortiz Pizarro²

PUYÉN, G. C.; ARMAS, P. J. \& ORTIZ, P. M. Efecto de una intervención educativa vía WhatsApp en la higiene oral de pacientes con ortodoncia. Int. J. Odontostomat., 14(4):575-580, 2020.

RESUMEN: La higiene oral en pacientes con ortodoncia fija se encuentra comprometida y las nuevas tecnologías de información podrían convertirse en una herramienta valiosa de educación en salud oral. El objetivo fue determinar el efecto de una intervención educativa vía whatsapp en la higiene oral de pacientes con aparatología de ortodoncia fija. La muestra del estudio estuvo conformada por 46 pacientes (18.65 \pm 3.21 años de edad) que iniciaron tratamiento de ortodoncia fija. Se realizó el índice de placa de O'Leary y se aplicó un cuestionario para medir el nivel de conocimiento en higiene oral. Los participantes se dividieron en un grupo experimental y control, mediante asignación aleatoria. Al grupo experimental se le envió una intervención educativa elaborada mediante la herramienta Play Ground Versión 2.0, vía la aplicación para mensajería instantánea WhatsApp Messenger Versión 2.19.153 y al grupo control se brindó las instrucciones de rutina sobre higiene oral. Después de 6 semanas, se evaluó el índice y cuestionario. Para el análisis de datos se utilizó el Coeficiente de Correlación de Concordancia, la prueba de Shapiro Wilk y la prueba T de Student para muestras relacionadas e independientes. Los pacientes del grupo experimental tuvieron diferencias estadísticamente significativas en el control de placa $(p=0.001)$ y nivel de conocimiento después de la intervención educativa $(p=0.016)$, a comparación del grupo control. La intervención educativa vía WhatsApp tuvo un efecto positivo en la higiene oral, con respecto al control de placa y al nivel de conocimientos de pacientes con ortodoncia fija.

PALABRAS CLAVE: ortodoncia, higiene oral, aplicación móvil.

\section{INTRODUCCIÓN}

El uso de aparatología de ortodoncia puede alterar sustantivamente el microambiente de la cavidad oral (Jing et al., 2019). Los pacientes con aparatología de ortodoncia fija son más susceptibles a contraer mayor placa bacteriana ya sea por las bandas, brackets, alambres, aumentando considerablemente el riesgo de contraer patologías periodontales como inflamación gingival, recesiones, formación de bolsas, hiperplasias y caries dental (Expósito Martín et al., 2010; Lara-Carrillo et al., 2010; Chen \& Zhou, 2015). El control efectivo de la placa y el cumplimiento de la higiene oral son desafíos importantes para el ortodoncista (Abdaljawwad, 2016; Kuppuswamy et al., 2014).

La falta de motivación y comunicación tiene un efecto negativo en el mantenimiento de la higiene oral (Mei et al., 2017). La evidencia científica ha demostra- do que los recordatorios activos son un mecanismo importante para producir cambios positivos en el comportamiento del paciente con respecto al tratamiento (Eppright et al., 2014; Lima et al., 2018). Según estudios, la educación y los mensajes recordatorios pueden mejorar efectivamente la higiene oral de los pacientes bajo tratamiento de ortodoncia y su conocimiento al respecto (Bowen et al., 2015; Cozzani et al., 2016; Li et al., 2016; Alkadhi et al., 2017).

Las redes sociales y las aplicaciones basadas en teléfonos inteligentes cambiaron la forma en que las personas interactúan con los sistemas de salud (Free et al., 2013; Santoro et al., 2015). En este sentido, WhatsApp es una aplicación gratuita, fácil de usar y que permite mensajería instantánea, compartir fotos, videos y audios. WhatsApp facilita todo tipo apren-

\footnotetext{
${ }^{1}$ Escuela de Odontología, Facultad de Medicina, Universidad Católica Santo Toribio de Mogrovejo, Chiclayo, Perú.

${ }^{2}$ Profesor adjunto, Escuela de Odontología, Facultad de Medicina, Universidad Católica Santo Toribio de Mogrovejo, Chiclayo, Perú.
} 
dizaje clínico y en el 2016 la base de usuarios alcanzó los mil millones (Boulos et al., 2016). En Latinoamérica, no existen estudios que midan la efectividad de una intervención educativa mediante herramientas tecnológicas de uso actual y masivo en pacientes bajo tratamiento de ortodoncia (Zotti et al., 2016).

La presente investigación tiene como propósito evaluar el efecto de una intervención educativa vía WhatsApp en la higiene oral de pacientes con aparatología de ortodoncia fija.

\section{MATERIAL Y MÉTODO}

El presente estudio fue aprobado y revisado de forma independiente por el Comité de Ética en Investigación de la Universidad Católica Santo Toribio de Mogrovejo, mediante resolución de aprobación número 630-2018-USAT-FMED. Todos los participantes, padres o tutores recibieron la explicación verbal y escrita con respecto a la investigación y brindaron su consentimiento de forma voluntaria.

Diseño del estudio. Se llevó a cabo una intervención educativa, controlado, aleatorio a doble ciego con dos brazos paralelos, con una proporción de asignación de la intervención educativa en 1: 1 para dos grupos de pacientes: control y experimental.

Criterios de selección. Se incluyeron pacientes de 13 a 23 años de edad, con diagnóstico de maloclusión Clase I, con un plan de tratamiento con aparatología fija multi-brackets y que tengan telefonía móvil tipo Smartphone con la aplicación WhatsApp activa.

Los pacientes con requerimiento inicial de aparatología adicional (arco transpalatino, lingual, entre otros), con ausencia de alguna pieza dentaria considerada en el índice de higiene oral, con signos de enfermedad periodontal clínicamente observable, que tomaban regularmente algún fármaco prescrito, con alteración de flujo salival, en estado gestacional o con antecedes de tratamiento ortodontico previo, fueron excluidos del estudio.

Muestra del estudio. La muestra inicial estuvo conformada por 79 pacientes, de los cuales después de evaluar los criterios de exclusión, quedaron 46 pacientes que iniciaban tratamiento de ortodoncia fija en tres consultorios privados de la ciudad de Chiclayo, Perú. Se seleccionó los pacientes mediante muestreo no probabilístico y se realizó una asignación aleatoria en dos grupos: 24 pacientes para el grupo control, 16 hombres y 8 mujeres (media 18,25 años SD 2,83) y 22 pacientes para el grupo experimental, 8 hombres y 14 mujeres (media 19,55 años SD 3,53). El tamaño de la muestra se calculó utilizando el software G*Power, versión 3.1.9. La potencia del estudio se estableció en 0.80 con un intervalo de confianza del $95 \%$ y el tamaño del efecto se estableció en 0,75.

Error del método. Se realizó un estudio piloto para la calibración en el método, validar el contenido de la intervención educativa y del cuestionario sobre higiene oral. Los investigadores se calibraron mediante la medición del índice de O'Leary en 10 participantes y comparando sus puntajes mediante el coeficiente de correlación de concordancia (CCC).

Procedimientos. El investigador principal evaluó a los pacientes para determinar la elegibilidad, explicar la naturaleza del estudio y obtener el obtener el consentimiento informado. Los pacientes que participaron en el estudio iniciaron tratamiento de ortodoncia con aparatología fija multi-brackets $(0,022 \times 0,028$ pulgadas, Mini Master, American Orthodontics, Sheboygan, WI, USA, técnica Roth), tubos adhesivos de la misma casa comercial en molares y mediante la técnica de cementación indirecta. Los pacientes en evaluación fueron atendidos por 2 especialistas en Ortodoncia, quienes coordinaron las instrucciones y procedimientos de higiene que se brindaron a todos los participantes.

Al inicio del tratamiento de ortodoncia, todos los pacientes recibieron instrucciones de higiene oral que se brindan de rutina y como parte del protocolo de atención se entregaron kits de higiene oral que contenían cepillo dental, cepillo interproximal, hilo dental y enguaje bucal. Después de 2 semanas se citó a los pacientes, se realizó el índice de placa de O'Leary y se aplicó un cuestionario con preguntas cerradas para evaluar el nivel de conocimiento en higiene oral. La validación del contenido del instrumento se realizó mediante el juicio de 3 expertos (AAN, CAM, MOP) y la confiabilidad del instrumento se evaluó mediante un coeficiente Alfa de Cronbach de 0,89. La asignación a los grupos experimental y control se realizó mediante números aleatorios con la correspondiente codificación para cada paciente de cada grupo y estuvo a cargo de un personal que no realizaba las mediciones del índice o la aplicación del cuestionario. Los resultados iniciales del índice de O'Leary y del cuestionario se mantuvieron en estricta reserva hasta el final de la intervención para mantener el ciego. 
Intervención educativa. Los pacientes del grupo experimental recibieron la intervención educativa a través de videos desarrollados utilizando la herramienta PlayGround Versión 2.0. El contenido de los videos educativos fue previamente validado en 10 pacientes no participantes del estudio, 1 especialista en ortodoncia, 1 especialista en periodoncia y 1 comunicador social. Los temas incluidos en la intervención educativa en orden fueron los siguientes: Importancia de la higiene oral en el tratamiento de ortodoncia, rol de la placa bacteriana, cepillo dental, pasta dental con flúor, hilo dental y enguaje bucal, así como técnicas de cepillado y de higiene oral.

Los videos educativos fueron enviados mediante la aplicación de mensajería para teléfonos inteligentes, WhatsApp Versión 2.19.153 o su última actualización y estuvo a cargo de un profesional en comunicaciones, de forma independiente y que no formaba parte del personal investigador. El programa educativo tuvo una duración de 6 semanas y fue enviado a los pacientes del grupo experimental de forma periódica. El grupo control mantuvo la educación motivacional de rutina brindada por los especialistas, la cual fue brindada a ambos grupos del estudio desde el inicio del tratamiento.

La intervención educativa fue estructurada en una etapa de inicio, proceso y salida. En la etapa de inicio, se enviaron dos videos durante la primera semana, con la finalidad de motivar al paciente, explicando la importancia de la higiene oral sobre su tratamiento. Al finalizar esta etapa, se envió una imagen que permitía al paciente valorar los videos educativos (me gustó/no me gustó) y verificar su participación.

La etapa de proceso se realizó en 4 semanas, se enviaron 4 videos, 1 por semana y en donde se les enseñó a los pacientes, las características de los aditamentos necesarios para su higiene oral y la técnica detallada de uso. Al finalizar esta etapa, se volvió a verificar la participación del paciente.

En la sexta semana, se llevó a cabo la etapa de cierre de la intervención mediante un video resumen con todo el contenido educativo además de volver a explicar la importancia de mantener la higiene oral en el éxito de su tratamiento. De igual manera, se envió una imagen que permitía al paciente valorar los videos y verificar su participación. Se citó 2 semanas después a los pacientes, se aplicó por segunda vez el cuestionario y se evaluó el índice de placa de O'Leary. Una vez concluida la intervención se autorizó el acceso a los puntajes iniciales del índice y del cuestionario.

Análisis estadístico. Para el procesamiento de los datos se utilizó el Programa Estadístico para las Ciencias Sociales (SPSS), versión 25.0. Para determinar el error del método intra e interexaminador con respecto al registro porcentual del índice de O'Leary, se utilizó el coeficiente de correlación de concordancia (CCC) con mediciones repetidas después de 2 semanas.

La confiabilidad o la homogeneidad de los ítems del cuestionario de conocimientos sobre higiene oral fue evaluada mediante el coeficiente Alfa de Cronbach. Se comprobó la normalidad de los datos mediante el test de Shapiro Wilk. Se utilizó la prueba T para muestras relacionadas para comparar dentro de un mismo grupo (antes y después de la intervención), mientras que la prueba $T$ para muestras independientes fue usada para comparar entre los dos grupos de estudio. El nivel de significancia considerado fue $p<0,05$.

\section{RESULTADOS}

La confiabilidad intra e interexaminador con respecto a la evaluación de la higiene oral se consideró adecuada. Se encontró alta concordancia con valores superiores a 0,93 (Tabla I).

Se observó que tanto el grupo experimental como el grupo control iniciaron en las mismas condiciones antes de la intervención educativa con respecto al índice de O'Leary, el grupo experimental con un valor porcentual de $74,31 \pm 8,87$ y el grupo control con $79,15 \pm 9.89 \%(p=0,232)$. Después de aplicar la intervención educativa vía Whatsapp, se observó una diferencia significativa $(p=0,001)$ entre los resultados

Tabla I. Confiabilidad intra e inter-examinador de la evaluación del índice de higiene oral (valores cuantitativos) [n=10]

\begin{tabular}{cccccccc}
\hline Variable & Calibration & CCC & SE & $95 \%$ Cl & Precision & Accuracy & $p$ value \\
\hline \multirow{2}{*}{ IHO } & Intra-examiner & 0,997 & 0,001 & {$[0,994-0,999]$} & 0,997 & 1,000 & $<0,001$ \\
& Inter-examiner & 0,995 & 0,002 & {$[0,991-0,999]$} & 0,996 & 0,999 & $<0,001$ \\
\hline
\end{tabular}

CCC: Coeficiente de Correlación de Concordancia; SE: Error Estándar; Cl: Intervalo Confidencial. 
del grupo experimental y grupo control, con valores de medias de 60,22 $\pm 12,086$ y $79,49 \pm 7,141$ respectivamente. (Tabla II).

En cuanto al nivel de conocimiento, ambos grupos iniciaron en las mismas condiciones antes de la intervención educativa. El puntaje del grupo experimental obtuvo una media de $6,92 \pm 1,83$ y el grupo control con una media de $8,27 \pm 1,95(p=0,101)$. Después de aplicar la intervención educativa vía WhatsApp, se observó una diferencia significativa ( $p$ $=0,016$ ) entre los puntajes del grupo experimental y

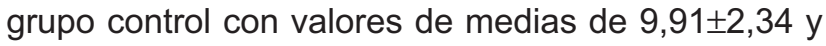
$7,50 \pm 2,02$ respectivamente (Tabla III).

En cuanto a la higiene oral, el grupo control inició en las mismas condiciones para hombres y mujeres $(p=0,438)$. De igual forma, en el grupo experi- mental tampoco hubo diferencias en la higiene oral con respecto al sexo $(p=0,568)$. Después de aplicar la intervención educativa vía WhatsApp, no se observó diferencia significativa entre hombres y mujeres del grupo control $(p=0,650)$. Sin embargo, sí se observó una mejora significativa en la higiene oral para las mujeres del grupo experimental con respecto a los hombres $(p=0,043)$ (Tabla IV).

En cuanto al nivel de conocimiento, en el grupo control, no existió una diferencia significativa entre hombres y mujeres antes de la intervención educativa $(p=0,398)$, de igual forma para el grupo experimental $(p=0,227)$. Después de aplicar la intervención educativa vía WhatsApp, tampoco se observó una diferencia significativa con respecto al sexo, tanto para el grupo control $(p=0,389)$ como en el grupo experimental $(p=0,264)($ Tabla V).

Tabla II. Efecto de una intervención educativa en el nivel de higiene oral de pacientes con aparatología de ortodoncia fija.

\begin{tabular}{|c|c|c|c|c|c|c|c|}
\hline \multirow{3}{*}{ MOMENTO } & \multicolumn{7}{|c|}{ GRUPO DE ESTUDIO } \\
\hline & & $\mathrm{col}$ & & & EXPER & TAL & n \\
\hline & $\mathrm{N}$ & Media & Desviación típ. & $\mathrm{N}$ & Media & Desviación típ. & p-valor(") \\
\hline IHO ANTES & 24 & 79,15 & 9,894 & 22 & 74,31 & 8,866 & 0,232 \\
\hline IHO DESPUES & 24 & 79,50 & 7,141 & 22 & 60,22 & 12,086 & 0,000 \\
\hline
\end{tabular}

(*) Prueba estadística T de Student.

Tabla III. Efecto de una intervención educativa en el nivel de conocimiento en pacientes con aparatología de ortodoncia fija.

\begin{tabular}{cccccccc}
\hline & \multicolumn{8}{c}{ GRUPO DE ESTUDIO } \\
NIVEL DE & \multicolumn{2}{c}{ CONTROL } & \multicolumn{2}{c}{ EXPERIMENTAL } & Media & Desviación típ. & p-valor $\left({ }^{*}\right)$ \\
CONOCIMIENTO & $\mathrm{N}$ & Media & Desviación típ. & $\mathrm{N}$ & Mediac & 0,101 \\
ANTES & 24 & 6,92 & 1,832 & 22 & 8,27 & 1,954 & 0,016 \\
DESPUES & 24 & 7,50 & 2,023 & 22 & 9,91 & 2,343 & 0,916 \\
\hline
\end{tabular}

$\left.{ }^{*}\right)$ Prueba estadística T de Student.

Tabla IV. Comparación del efecto de una intervención educativa en el nivel de higiene oral de pacientes con aparatología de ortodoncia fija, según sexo.

\begin{tabular}{|c|c|c|c|c|c|c|}
\hline \multirow{2}{*}{$\mathrm{IHO}$} & \multicolumn{3}{|c|}{ CONTROL } & \multicolumn{3}{|c|}{ EXPERIMENTAL } \\
\hline & $\mathrm{F}$ & $\mathrm{M}$ & p-valor & $\mathrm{F}$ & $\mathrm{M}$ & $\mathrm{p}$-valor $\left(^{*}\right)$ \\
\hline ANTES & $82,51 \pm 10.38$ & $77,47 \pm 9.90$ & 0,438 & $75,55 \pm 7,09$ & $72,15 \pm 12,31$ & 0,568 \\
\hline DESPUES & $\begin{array}{c}80,91 \pm 8.51 \\
n=8\end{array}$ & $\begin{array}{c}78,79 \pm 6.88 \\
n=16\end{array}$ & 0,650 & $\begin{array}{c}54,84 \pm 9,52 \\
n=14\end{array}$ & $\begin{array}{c}69,64 \pm 10,94 \\
n=8\end{array}$ & 0,043 \\
\hline
\end{tabular}

(*) Prueba estadística T de Student.

Tabla V. Comparación del efecto de una intervención educativa en el nivel de conocimiento en pacientes con aparatología de ortodoncia fija, según sexo.

\begin{tabular}{lcccccc}
\hline \multicolumn{1}{c}{ NIVEL DE } & & CONTROL & & \multicolumn{3}{c}{ EXPERIMENTAL } \\
\multicolumn{1}{c}{ CONOCIMIENTO } & $\mathrm{F}$ & $\mathrm{M}$ & $\mathrm{p}$-valor & $\mathrm{F}$ & $\mathrm{M}$ & $\mathrm{p}$-valor( $\left.{ }^{*}\right)$ \\
\hline ANTES & $6,25 \pm 0,96$ & $7,25 \pm 2,12$ & 0,3983 & $7,71 \pm 1,80$ & $9,25 \pm 2,06$ & 0,2273 \\
DESPUES & $6,75 \pm 1,26$ & $7,875 \pm 2,30$ & 0,3891 & $9,29 \pm 2,14$ & $11,00 \pm 2,58$ & 0,264 \\
& $\mathrm{n}=8$ & $\mathrm{n}=16$ & & $\mathrm{n}=14$ & $\mathrm{n}=8$ & 8 \\
\hline
\end{tabular}

(*) Prueba estadística T de Student. 


\section{DISCUSIÓN}

Lima et al., a través de una revisión sistemática y un meta análisis, han señalado la importancia de lograr una adecuada motivación con respecto a la higiene oral en pacientes con tratamiento de ortodoncia fija y el impacto que podría tener en la reducción de placa y de otras patologías asociadas. El presente estudio utilizó plataformas digitales como PlayGround y la aplicación de mensajería instantánea WhatsApp, los cuales han demostrado ser herramientas actuales y útiles para lograr la modificación de patrones de comportamiento, actitudes y conocimientos (Woodcock et al., 2012; Zomeño \& Blay-Arráez, 2017), en pacientes que hicieron uso constante de telefonía móvil tipo Smartphone durante el estudio.

El presente estudio estuvo conformado por dos grupos de participantes a quienes se les realizó una intervención educativa de seis semanas de duración y los resultados se midieron utilizando el índice de O'Leary y un cuestionario de conocimientos. Según los resultados del presente estudio se pudo observar que se logró estandarizar a los pacientes en cuanto a su índice de placa bacteriana y nivel de conocimientos antes de aplicar la intervención educativa, evidenciando que el grupo experimental y control era comparables. A partir de los resultados obtenidos, los valores muestran que la higiene oral y el nivel de conocimientos de los pacientes del grupo experimental, obtuvo una mejora significativa con respecto a los pacientes del grupo control, siendo las mujeres las que presentaron un mejor control de placa.

La mayoría de estudios disponibles han utilizado mensajes de texto o llamadas telefónicas como una forma de recordatorio con buenos resultados, pero sólo tres estudios hasta el momento han incorporado las nuevas plataformas digitales de comunicación y aplicaciones móviles como una forma de acompañamiento que permita mantener o mejorar la higiene oral en pacientes con aparatología ortodontica. Alkadhi et al., en un ensayo controlado y aleatorizado, midieron el índice de placa (IP) y el índice gingival (IG) obteniendo una mejoría significativa en los pacientes después de recibir recordatorios de higiene oral a través de una aplicación móvil durante un período de estudio de cuatro semanas. Por otro lado, en un ensayo clínico llevado a cabo por Li et al., durante un año y nueve meses, no se encontró diferencias significativas entre el grupo control y experimental con respecto al índice de placa (IP) y al índice gingival modificado. Finalmente,
Zotti et al. evaluaron el índice gingival e índice de placa cada tres meses durante un año, en un ensayo clínico a un grupo control y experimental, no encontrando diferencias significativas al final del tercer mes de seguimiento, pero observando diferencias significativas en el resto de mediciones posteriores.

En el presente estudio se utilizó el índice de O'Leary para evaluar el control de placa, mediante el cual se busca demostrar el principio de autocuidado por parte del paciente de ortodoncia, ofreciendo ciertas cualidades como instrumento de medición gracias a una validez universal y sensibilidad diagnóstica mayor frente a otros índices de detección de placa (Corchuelo, 2011). Sin embargo, también tiene algunas características que dificultad su ejecución, como requerir todas las piezas presentes (no se permite el remplazo) y un mayor tiempo de evaluación. Los resultados con respecto a una mejora en la higiene oral gracias a la intervención coinciden con dos de los estudios disponibles, a pesar de las diferencias en los criterios de exclusión, tamaño de muestra y en el número de mediciones. Si bien un tamaño mayor hubiera sido deseable dado que ayuda a evidenciar una mayor potencia estadística del estudio, en este caso, las diferencias se lograron establecer desde un menor número de participantes (Jones et al., 2003).

Una consideración importante a partir de los resultados, es la necesidad de probar nuevos contenidos educativos que puedan lograr un adecuado control de placa y que puedan ser utilizados en más y nuevas plataformas de comunicación, dentro de estudios con muestras y periodos de seguimiento mayores. Se concluye que la intervención educativa vía WhatsApp tuvo en efecto positivo en el nivel de higiene con respecto al control de placa y al nivel de conocimientos en pacientes con ortodoncia fija, con una mejora significativamente mayor en las mujeres.

PUYÉN, G. C.; ARMAS, P. J. \& ORTIZ, P. M. Effect of an educational intervention via WhatsApp on the oral hygiene of orthodontic patients. Int. J. Odontostomat., 14(4):575-580, 2020.

ABSTRACT: Oral hygiene in patients with fixed orthodontics is compromised and new information technologies could become a valuable tool for oral health education. The objective of the study was to determine the effect of an educational intervention via WhatsApp on the oral hygiene of patients with fixed orthodontic appliances. The study sample consisted of 46 patients $(18.65 \pm 3.21$ years old) who started fixed orthodontic treatment. The O'Leary 
plaque index was performed and a questionnaire was applied to measure the level of knowledge in oral hygiene. Participants were divided into an experimental and control group, by randomization. The experimental group was sent an educational intervention prepared using the Play Ground Version 2.0 tool, via the WhatsApp Messenger version 2.19.153 instant messaging application, and the control group was given routine instructions on oral hygiene. After 6 weeks, the index and questionnaire were evaluated. For data analysis, the concordance correlation coefficient, the Shapiro Wilk test and the Student's T test for related and independent samples were used. The patients in the experimental group had statistically significant differences in plaque control $(p=$ $0.001)$ and level of knowledge after the educational intervention ( $p=0.016$ ), compared to the control group. The educational intervention via WhatsApp had a positive effect on oral hygiene, with respect to plaque control and the level of knowledge of patients with fixed orthodontics.

KEY WORDS: orthodontics, oral hygiene, mobile application.

\section{REFERENCIAS BIBLIOGRÁFICAS}

Abdaljawwad, A. The influence of text message reminders on oral hygiene compliance in orthodontic patients. Iraqi. Dent. J., 38(1):58-62, 2016.

Alkadhi, O. H.; Zahid, M. N.; Almanea, R. S.; Althaqeb, H. K.; Alharbi, T. H. \& Ajwa, N. M. The effect of using mobile applications for improving oral hygiene in patients with orthodontic fixed appliances: a randomised controlled trial. J. Orthod., 44(3):15763, 2017.

Boulos, M. N. K.; Giustini, D. M. \& Wheeler, S. Instagram and WhatsApp in health and healthcare: an overview. Future Internet, 8(3):37, 2016.

Bowen, T. B.; Rinchuse, D. J.; Zullo, T. \& DeMaria, M. E. The influence of text messaging on oral hygiene effectiveness. Angle Orthod., 85(4):543-8, 2015.

Corchuelo, J. Sensibilidad y especificidad de un índice de higiene oral de uso comunitario. Colomb. Med., 42(4):448-57, 2011.

Cozzani, M.; Ragazzini, G.; Delucchi, A.; Mutinelli, S.; Barreca, C.; Rinchuse, D. J.; Servetto, R. \& Piras, V. Oral hygiene compliance in orthodontic patients: a randomized controlled study on the effects of a post-treatment communication. Prog. Orthod., 17(1):41, 2016.

Chen, W. \& Zhou, Y. Caries outcomes after orthodontic treatment with fixed appliances: a longitudinal prospective study. Int. J. Clin. Exp. Med., 8(2):2815-22, 2015.

Eppright, M.; Shroff, B.; Best, A. M.; Barcoma, E. \& Lindauer, S. J. Influence of active reminders on oral hygiene compliance in orthodontic patients. Angle Orthod., 84(2):208-13, 2014.

Expósito Martín, M.; Cuan Corrales, M.; Estrada Verdeja, V. \& Martín Zaldívar, L. Factores de riesgo a caries en pacientes con aparatos ortodónticos fijos. A. M. C., 14(5):1-11, 2010.

Free, C.; Phillips, G.; Watson, L.; Galli, L.; Felix, L.; Edwards, P.; Patel, V. \& Haines, A. The effectiveness of mobile-health technologies to improve health care service delivery processes: a systematic review and meta-analysis. PLoS Med., 10(1):e1001363, 2013.
Jing, D.; Hao, J.; Shen, Y.; Tang, G.; Lei, L. \& Zhao, Z. Effect of fixed orthodontic treatment on oral microbiota and salivary proteins. Exp. Ther. Med., 17(5):4237-43, 2019.

Jones, S.; Carley, S. \& Harrison, M. An introduction to power and sample size estimation. Emerg. Med. J., 20(5):453-8, 2003.

Kuppuswamy, V. L.; Murthy, S.; Sharma, S.; Surapaneni, K. M.; Grover, A. \& Joshi, A. Oral hygiene status, knowledge, perceptions and practices among school settings in rural South India. Oral Health Dent. Manag., 13(1):146-54, 2014.

Lara-Carrillo, E.; Montiel-Bastida, N. M.; Sánchez-Pérez, L. \& AlanísTavira, J. Effect of orthodontic treatment on saliva, plaque and the levels of Streptococcus mutans and Lactobacillus. Med. Oral Patol. Oral Cir. Bucal, 15(6):e924-9, 2010.

Li, X.; Xu, Z. R.; Tang, N.; Ye, C.; Zhu, X. L.; Zhou, T. \& Zhao, Z. H. Effect of intervention using a messaging app on compliance and duration of treatment in orthodontic patients. Clin. Oral Investig., 20(8):1849-59, 2016.

Lima, I. F. P.; de Andrade Vieira, W.; de Macedo Bernardino, Í.; Costa, P. A.; Lima, A. P. B.; Pithon, M. M. \& Paranhos, L. R. Influence of reminder therapy for controlling bacterial plaque in patients undergoing orthodontic treatment: A systematic review and metaanalysis. Angle Orthod., 88(4):483-93, 2018.

Mei, L.; Chieng, J.; Wong, C.; Benic, G. \& Farella, M. Factors affecting dental biofilm in patients wearing fixed orthodontic appliances. Prog. Orthod., 18:4, 2017.

Santoro, E.; Castelnuovo, G.; Zoppis, I.; Mauri, G. \& Sicurello, F. Social media and mobile applications in chronic disease prevention and management. Front. Psychol., 6:567, 2015.

Woodcock, B.; Middleton, A. \& Nortcliffe, A. Considering the smartphone learner: An investigation into student interest in the use of personal technology to enhance their learning. Stud. Engagem. Exp. J., 1(1):1-15, 2012.

Zomeño, D. \& Blay-Arráez, R. Diseño de formatos informativos en las redes sociales dirigidos al público millennial. El caso del Videonews de PlayGround. Prof. Inf., 26(6):1184-91, 2017.

Zotti, F.; Dalessandri, D.; Salgarello, S.; Bonetti, S.; Visconti, L. \& Paganelli, C. Usefulness of an app in improving oral hygiene compliance in adolescent orthodontic patients. Angle Orthod., 86(1):101-7, 2016.

Dirección para correspondencia a:

Mariano Ortiz Pizarro

Universidad Católica Santo Toribio de Mogrovejo PERÚ

Email: mortiz@usat.edu.pe

Recibido : 08-05-2020

Aceptado: 11-07-2020 